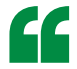

...if SpoVM were sensing the positive curvature of the forespore membrane, it should bind to any phospholipid bilayer with a similar curvature.

\section{BACTERIAL PHYSIOLOGY}

\section{Ahead of the curve}

Protein localization within a bacterial cell can rely on a range of factors, such as signal sequences and interacting partners. Ramamurthi et al. now add detection of membrane curvature to the list, with their demonstration, published in Science, that recruitment of the Bacillus subtilis spore coat protein SpoVM to the forespore membrane depends on its ability to sense the positive curvature of the bilayer.

During spore formation, B. subtilis divides asymmetrically to create a mother cell and a forespore compartment. The forespore is then engulfed by the mother cell, producing a double-membraned cell-within-acell, onto which a proteinaceous coat is deposited. Spore coat formation requires SpoVM, a small, amphipathic, helical peptide that inserts into the membrane with its long axis parallel to the plane of the membrane and its hydrophobic face buried in the lipid bilayer. GFP-labelled SpoVM (SpoVM-GFP) localizes specifically to the forespore membrane in a manner that is dependent on proline 9; substitution of this amino acid for alanine (P9A SpoVM-GFP) decreases the specific localization to the forespore membrane, allowing binding to the cytoplasmic membrane of the mother cell.

The authors proposed that geometric cues might be responsible for the specificity of SpoVM localization, as the forespore membrane displays positive curvature, whereas the cytoplasmic membrane is negatively curved. To test this theory, the authors used a mutant strain of B. subtilis in Massachusetts, USA.

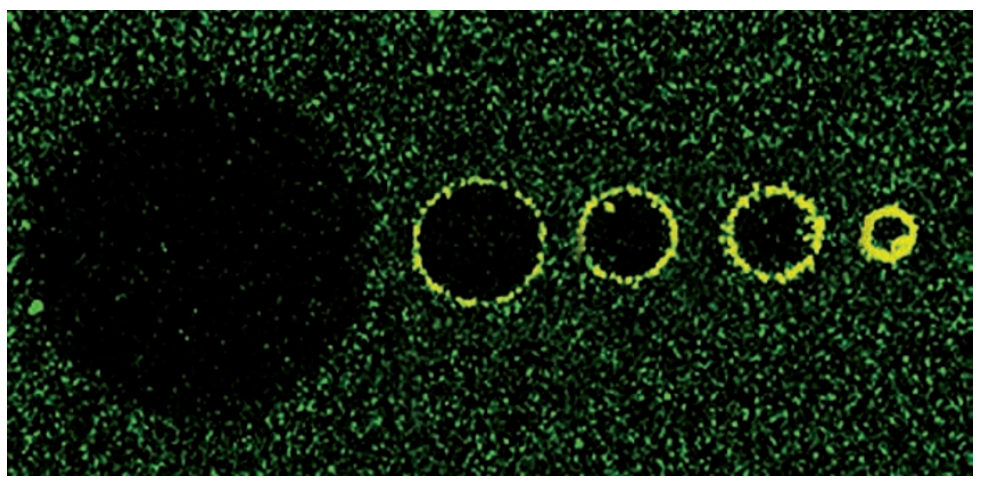

Confocal light microscopy image of SpoVM incubated with a heterogeneous population of unilamellar phospholipid vesicles. Image courtesy of S. Lecuyer and K. Ramamurthi, Harvard University,

which spore formation arrests prior to engulfment, leaving a straight polar septum between the mother and forespore. SpoVM-GFP showed no preference in binding the septum or cytoplasmic membrane. Ramamurthi and colleagues reasoned that if SpoVM were sensing the positive curvature of the forespore membrane, it should bind to any phospholipid bilayer with a similar curvature. The authors expressed SpoVM-GFP in strains of Escherichia coli and Saccharomyces cerevisiae that contained internal vesicles of a similar size to the B. subtilis forespore. SpoVMGFP specifically labelled these vesicles, whereas P9A SpoVM-GFP labelled cytoplasmic membranes as well. In vitro, they found that purified SpoVM-GFP preferentially bound to the smallest (and therefore more convex) vesicles in a heterogeneous population of unilamellar vesicles.

For a rod-like molecule $40 \AA$ in length, such as SpoVM, the distance between one end of the rod and the surface of the bilayer can be a maximum of $0.2 \AA$, making it improbable that a single molecule of SpoVM is able to sense the curve of the membrane alone. The authors found that for vesicle diameters of about $4 \mu \mathrm{m}$ or less, adsorption of SpoVM-GFP peptides increases as a function of decreasing membrane curvature and increasing peptide concentration, suggesting that cooperative clustering of SpoVM occurs. Preliminary analysis suggests that this is likely to involve only a few SpoVM molecules; however, individual molecules of SpoVM do not seem to interact with each other, so the exact nature of this cooperation remains to be determined.

Andrew Jermy

ORIGINAL RESEARCH PAPER Ramamurthi, K. S. et al. Geometric cue for protein localization in a bacterium. Science 323, 1354-1357 (2009) 